International Journal of Modern Physics C Vol. 27, No. 12 (2016) 1699001 (13 pages) (C) World Scientific Publishing Company DOI: $10.1142 / \mathrm{S} 0129183116990011$

\title{
Author Index (Volume 27)
}

Abdel Aal, S., Reactivity of boron-and nitrogen-doped carbon nanotubes functionalized by (Pt, Eu) atoms toward $\mathrm{O}_{2}$ and $\mathrm{CO}: \mathrm{A}$ density functional study

Abidri, B., see Labair, M.

Achahbar, A., see Lachgar, A.

Afify, A. A., see El-Aziz, M. A.

Aguilar-López, R., LópezPérez, P. A., LaraCisneros, G. \& Femat, R., Controlling a class of chaotic quantum system under disturbances and noisy measurements: Application to $1 D$ BoseEinstein condensate

Ahmed, R., see Labair, M. Alba, A., Mendez, M. O., Rubio-Rincon, M. E. \& Arce-Santana, E. R., $A$ consensus algorithm for approximate string matching and its application to QRS complex detection

Alba, A., see De LeónLomelí, R.

Alba, A., see ReyesSanchez, E.

Alvarado-Méndez, E., see Coronel-Escamilla, A.

Álvarez, G., see ZavalaGuillén, I.

Andrade Jr., J. S., see de Brito, J. B.

Andrade, J. S., see Verma, T.

Andrecut, M., Local operators in kinetic wealth distribution

Andrecut, M., Systemic risk, maximum entropy and interbank contagion

\section{7 (2016) 1650075}

9 (2016) 1650107

2 (2016) 1650020

12 (2016) 1650150

4 (2016) 1650040

9 (2016) 1650107

3 (2016) 1650029

7 (2016) 1650071

12 (2016) 1650140

8 (2016) 1650084

8 (2016) 1650095

3 (2016) 1650024

12 (2016) 1650141

11 (2016) 1650132

12 (2016) 1650148
Araújo, N. A. M., see Verma, $\mathrm{T}$.

Arce, J., see ZavalaGuillén, I.

Arce-Santana, E. R., see Alba, A.

Arslan, H., see Taran, S.

Ashrafizaadeh, M., see Zadehgol, A.

Azevedo, S. M., Saba, H., Miranda, J. G. V., Filho, A. S. N. \& Moret, M. A., Self-affinity in the dengue fever time series

Bagli, E. \& Karpusenko, V., Exploiting $M I C$ architectures for the simulation of channeling of charged particles in crystals

Baiocco, S., see Bevilacqua, A.

Bella, G., see Di Ilio, G.

Benalia, S., see Labair, M.

Benavides-Cruz, M., see Gómez-Aguilar, J. F.

Benavides-Cruz, M., Calderón-Ramón, C., Gomez-Aguilar, J. F., Rodríguez-Achach, M., Cruz-Orduña, I., Laguna-Camacho, J. R., Morales-Mendoza, L. J., Enciso-Aguilar, M., Pérez-Meana, H., Escalante-Martínez, J. E., López-Calderón, J. E. \& Juárez-Morales, G., Numerical simulation of metallic nanostructures interacting with electromagnetic fields using the Lorentz-Drude model and FDTD method
12 (2016) 1650141

8 (2016) 1650095

3 (2016) 1650029

12 (2016) 1650146

8 (2016) 1650088

12 (2016) 1650143

2 (2016) 1650022

9 (2016) 1650106

12 (2016) 1650139

9 (2016) 1650107

1 (2016) 1650007

4 (2016) 1650043 
Bentaleb, K., Lakouari, N., Ez-Zahraouy, H. \& Benyoussef, A., Simulation study of satisfaction rate in the mixed traffic flow with open boundary conditions

Benyoussef, A., see Bentaleb, K.

Benyoussef, A., see Echab, H.

Benyoussef, A., see Lamzabi, S.

Bera, R. K., see Sahoo, S.

Bertotti, M. L. \& Modanese, G., Microscopic models for the study of taxpayer audit effects

Bevilacqua, A. \& Baiocco, S., Automatic classification of lung tumour heterogeneity according to a visual-based score system in dynamic contrast enhanced CT sequences

Bhowmick, S. K. \& Ghosh, D., Targeting engineering synchronization in chaotic systems

Bo, L., see Heng, Z.

Bouhemadou, A., see Labair, M.

Braga, J. P. M. \& Filho, R. N. C., Nonadditive quantum mechanics as a Sturm-Liouville problem Bulut, F., see Oruç, Ö.

Bustos-Guajardo, R. \& Moukarzel, C. F., Wealth distribution under Yard-Sale exchange with proportional taxes

Cai, X., see Su, Z.

Cai, X., see Zhu, Y.

Cai, X., see Zou, Y.

Calderón-Ramón, C., see Benavides-Cruz, M.

Calderón-Ramón, C., see Gómez-Aguilar, J. F.

Camarano, A., see Sergi, D.

Campos-Cantón, E., Chaotic attractors based on unstable dissipative systems via third-order differential equation
2 (2016) 1650023

2 (2016) 1650023

1 (2016) 1650009

6 (2016) 1650068

7 (2016) 1650074

9 (2016) 1650100

9 (2016) 1650106

1 (2016) 1650006

1 (2016) 1650005

9 (2016) 1650107

4 (2016) 1650047

9 (2016) 1650103

8 (2016) 1650094

12 (2016) 1650147

10 (2016) 1650122

8 (2016) 1650090

4 (2016) 1650043

1 (2016) 1650007

6 (2016) 1650062

1 (2016) 1650008
Canto-Lugo, E., see Rodríguez-Achach, M.

Chai, Y., see Liu, S.

Chang, B., see Liu, D.

Chang, W., see Ko, B.

Chen, C., see Han, D.

Chen, L., see Li, Z.

Chen, L.-Q., see Liu, S.

Chen, Q., Zhang, X. B., Li, Q., Jiang, X. S. \& Zhou, H. P., Study of three-dimensional electroosmotic flow with curved boundary via lattice Boltzmann method

Chen, S., see $\mathrm{Li}, \mathrm{Z}$.

Cheng, R.-J., see Liu, Y.

Cherubini, C., see Nestola, M. G. C.

Chiappini, D., see Di Ilio, G.

Chiccoli, C., Pasini, P., Evangelista, L. R., De Souza, R. T. \& Zannoni, C., Field effects on inversion walls in nematic films: A computer simulation study

Chouvarda, I., see De León-Lomelí, R.

Coronel-Brizio, H. F., see Rodríguez-Achach, M.

Coronel-Escamilla, A., Gómez-Aguilar, J. F., Alvarado-Méndez, E., Guerrero-Ramírez, G. V. \& Escobar-Jiménez, R. F., Fractional dynamics of charged particles in magnetic fields

Couceiro, M., see Gama, J.

Crokidakis, N., Noise and disorder: Phase transitions and universality in a model of opinion formation

Cruz-Orduña, I., see Benavides-Cruz, $M$.

Das, S., see Sahoo, S.

Davids, K., see Gama, J. de Brito, J. B., Sampaio Filho, C. I. N., Moreira, A. A. \& Andrade Jr., J. S., Characterizing the intrinsic correlations of scale-free networks
11 (2016) 1650127

11 (2016) 1650129

9 (2016) 1650105

4 (2016) 1650038

6 (2016) 1650057

3 (2016) 1650034

11 (2016) 1650129

6 (2016) 1650063

6 (2016) 1650066

10 (2016) 1650111

2 (2016) 1650017

12 (2016) 1650139

10 (2016) 1650114

7 (2016) 1650071

11 (2016) 1650127

8 (2016) 1650084

9 (2016) 1650102

6 (2016) 1650060

4 (2016) 1650043

7 (2016) 1650074

9 (2016) 1650102

3 (2016) 1650024 
Author Index

De León-Lomelí, R., Murguía, J. S., Chouvarda, I., Méndez, M. O., González-Galván, E. \& Alba, A., Scaling analysis of heart beat fluctuations data and its relationship with cyclic alternating pattern data during sleep

De Martino, D., The dual of the space of interactions in neural network models

De Oliveira, P. P. B., see Ruivo, E. L. P.

De Souza, R. T., see Chiccoli, C.

Deng, W., see $\mathrm{Su}, \mathrm{Z}$.

Deng, W., see Zou, Y.

Deng, Y., see Zhang, Q.

Dereli, Y., see Korkmaz, B.

Deyranlou, A., Niazmand, H., Sadeghi, M.-R. \& Mesri, Y., Non-newtonian effects of blood on LDL transport inside the arterial lumen and across multi-layered arterial wall with and without stenosis

Di Ilio, G., Chiappini, D. \& Bella, G., A comparison of numerical methods for non-Newtonian fluid flows in a sudden expansion

Dias, G., see Gama, J.

Ding, H., see Liu, S.

Ding, J.-X., see Wang, J.

Ding, Z.-J., see Sun, X.-Y.

Dion, C. M., see Hashemloo, A.

Do, Y., see Zheng, M.

Dorso, C. O., see Frank, G. A.

Du, R., see Wu, J.

Du, W.-B., see Hong, C.

Echab, H., Lakouari, N., Ez-Zahraouy, H. \& Benyoussef, A., Simulation study of traffic car accidents at a single lane roundabout
7 (2016) 1650071

6 (2016) 1650067

7 (2016) 1650073

10 (2016) 1650114

12 (2016) 1650147

8 (2016) 1650090

10 (2016) 1650118

10 (2016) 1650117

1 (2016) 1650003

12 (2016) 1650139

9 (2016) 1650102

11 (2016) 1650129

2 (2016) 1650013

6 (2016) 1650058

2 (2016) 1650014

5 (2016) 1650048

8 (2016) 1650091

8 (2016) 1650092

11 (2016) 1650131

1 (2016) 1650009
El-Aziz, M. A. \& Afify, A. A., Lie group analysis of hydromagnetic flow and heat transfer of a powerlaw fluid over stretching surface with temperaturedependent viscosity and thermal conductivity

Elbitar, A. M., see Radwan, M. A.

Encinas, A. H., GayosoMartínez, V., Martín del Rey, A., MartínVaquero, J. \& Queiruga-Dios, A., A study on the efficiency and stability of high-order numerical methods for Form-II and Form-III of the nonlinear KleinGordon equations

Enciso-Aguilar, M., see Benavides-Cruz, M.

Enjilela, V., Salimi, D., Tavasoli, A. \& Lotfi, M., Stabilized MLPG$V F$-based method with $C B S$ scheme for laminar flow at high Reynolds and Rayleigh numbers

Escalante-Martínez, J. E., see Benavides-Cruz, M.

Escobar-Jiménez, R. F., see Coronel-Escamilla, A.

Escobar-Jiménez, R. F., see Gómez-Aguilar, J. F.

Esen, A., see Oruç, Ö.

Evangelista, L. R., see Chiccoli, C.

Ez-Zahraouy, H., see Bentaleb, $\mathrm{K}$

Ez-Zahraouy, H., see Echab, $\mathrm{H}$.

Ez-Zahraouy, H., see Lamzabi, S.

Femat, R., see AguilarLópez, R.

Feng, G., see Gao, X.

Feng, X. \& Hu, H., CCP interoperability and system stability

Filho, A. S. N., see Azevedo, S. M.
12 (2016) 1650150

9 (2016) 1650109

9 (2016) 1650097

4 (2016) 1650043

7 (2016) 1650081

4 (2016) 1650043

8 (2016) 1650084

1 (2016) 1650007

9 (2016) 1650103

10 (2016) 1650114

2 (2016) 1650023

1 (2016) 1650009

6 (2016) 1650068

4 (2016) 1650040

11 (2016) 1650138

3 (2016) 1650025

12 (2016) 1650143 
Filho, R. N. C., see Braga, J. P. M.

Filippi, S., see Nestola, M. G. C.

Frank, G. A. \& Dorso, C. O., Panic evacuation of single pedestrians and couples

$\mathrm{Fu}$, B.-B., see Zhang, L.

$\mathrm{Fu}$, C.-J., see Jia, X.

Gama, J., Dias, G., Couceiro, M., Passos, P., Davids, K. \& Ribeiro, J., An ecological dynamics rationale to explain home advantage in professional football

Gan, L., see Gong, X.

Gao, X., Ren, L., Shang, P. \& Feng, G., The scaling of time series size towards detrended fluctuation analysis

Gao, Y.-C., see Jia, X.

Gao, Z. Y., see Qiang, S. J.

Garip, A. K., see Taran, $\mathrm{S}$.

Gayoso-Martínez, V., see Encinas, A. H.

Ge, H.-X., see Liu, Y.

Ghazanfarian, J., Saghatchi, R. \& GorjiBandpy, M., SPH simulation of turbulent flow past a high-frequency inline oscillating cylinder near free-surface

Ghosh, D., see Bhowmick, S. K.

Gijón-Rivera, M., see Zavala-Guillén, I.

Gill, W., Computer simulation for the growing probability of additional offspring with an advantageous reversal allele in the decoupled continuoustime mutation-selection model

Gizzi, A., see Nestola, M. G. C.

Gomez-Aguilar, J. F., see Benavides-Cruz, M.
4 (2016) 1650047

2 (2016) 1650017

8 (2016) 1650091

12 (2016) 1650145

7 (2016) 1650082

9 (2016) 1650102

1 (2016) 1650010

11 (2016) 1650138

7 (2016) 1650082

6 (2016) 1650061

12 (2016) 1650146

9 (2016) 1650097

10 (2016) 1650111

12 (2016) 1650152

1 (2016) 1650006

8 (2016) 1650095

6 (2016) 1650070

2 (2016) 1650017

4 (2016) 1650043
Gómez-Aguilar, J. F., Escobar-Jiménez, R. F., Olivares-Peregrino, $\mathrm{V}$. H., Benavides-Cruz, M. \& Calderón-Ramón, C., Nonlocal electrical diffusion equation

1 (2016) 1650007

Gómez-Aguilar, J. F., see Coronel-Escamilla, A.

Gong, X., Gan, L. \& Wu, Z., Adaptive impulsive cluster synchronization in community network with nonidentical nodes

González-Galván, E., see De León-Lomelí, R.

Gorji-Bandpy, M., see Ghazanfarian, J.

Górski, A. Z., Stróż, M., Ośw ięcimka, P. \& Skrzat, J., Accuracy of the box-counting algorithm for noisy fractals

Gu, B., see Zhang, X.-J.

Guan, J. \& Wang, K., Consistent evolution in a pedestrian flow

Guan, W., see Huang, A.

Guan, X.-M., see Zhang, X.-J.

Guerrero-Ramírez, G. V., see Coronel-Escamilla, A.

Gündüz, G., The dynamics of the rise and fall of empires

Guo, Q., see Ma, J.

Guo, Q., see Ma, J.

Guo, Q., see Ma, J.

Guo, Q., see Tang, L.-Y.

Hadzibeganovic, T., see Lichtenegger, K.

Han, D., Li, D., Chen, C. \& Sun, M., How the heterogeneous infection rate effect on the epidemic spreading in activity-driven network

Han, J., see $\mathrm{Su}, \mathrm{Z}$.

Han, M., Zhang, Y. \& Zhang, M., Modified function projective synchronization between two different complex networks with delayed couplings and delayed nodes of different dimensions
8 (2016) 1650084

1 (2016) 1650010

7 (2016) 1650071

12 (2016) 1650152

10 (2016) 1650112

8 (2016) 1650093

9 (2016) 1650104

6 (2016) 1650064

8 (2016) 1650093

8 (2016) 1650084

10 (2016) 1650123

3 (2016) 1650028

4 (2016) 1650044

5 (2016) 1650054

4 (2016) 1650046

5 (2016) 1650065

6 (2016) 1650057

12 (2016) 1650147

11 (2016) 1650126 
Han, W., see Ma, J.

Han, W., see $\mathrm{Ma}$, J.

Han, W., see Ma, J.

Harfash, A. J., Stability analysis for penetrative convection in a fluid layer with throughflow

Hashemian, M., see Zarei, M.

Hashemloo, A., Dion, C. M. \& Rahali, G., Wave packet dynamics of an atomic ion in a Paul trap: approximations and stability

$\mathrm{He}$, J., see Li, S.

He, X., Niu, K., He, Z., Lin, J., Zhang, H. \& Jiang, Z.-Y., Efficient packet navigation method on scale-free networks with finite and diversiform node delivery capacity

$\mathrm{He}, \mathrm{Z}$., see $\mathrm{He}, \mathrm{X}$.

Heng, Z., Xiao, X., Bo, L., Jin, W. W., Yi, H. \& Youqin, W., Theoretical investigation of a tunable external cavity diode laser based on a single cavity all-dielectric thinfilm Fabry-Perot filter

Hernández-Montoya, A. R., see RodríguezAchach, M.

Hernández-Pérez, I., see Zavala-Guillén, I.

Herrmann, H. J., see Verma, $\mathrm{T}$.

Higuchi, S., see Kimura, K.

Hong, C., Zhang, J., Du, W.-B., Sallan, J. M. \& Lordan, O., Cascading failures with local load redistribution in interdependent Watts-Strogatz networks

Hong, J.-S., see Jia, X.

Hongxia, G., see Pengjun, $\mathrm{Z}$.

Hopstad, Y. \& Myrheim, J., Computer simulations of rotating systems of few particles bound by gravitation
4 (2016) 1650044

5 (2016) 1650054

3 (2016) 1650028

9 (2016) 1650101

10 (2016) 1650119

2 (2016) 1650014

10 (2016) 1650121

9 (2016) 1650098

9 (2016) 1650098

1 (2016) 1650005

11 (2016) 1650127

8 (2016) 1650095

12 (2016) 1650141

11 (2016) 1650128

11 (2016) 1650131

7 (2016) 1650082

11 (2016) 1650135

12 (2016) 1650142
Hou, L., see Yan, H.

Hou, S., see Huang, S.

$\mathrm{Hu}, \mathrm{H}$., see Feng, $\mathrm{X}$.

Hu, J.-Q., see Jia, X.

$\mathrm{Hu}, \mathrm{M} .-\mathrm{B}$., see Wang, D. $-X$.

Hu, M.-B., see Zhang, H.$\mathrm{Z}$.

Huang, A., Xiong, J., Shen, J. \& Guan, W., Evolution of weighted complex bus transit networks with flow

Huang, G., Zhang, P., Zhang, B., Yin, T. \& Ren, J., The optimal community detection of software based on complex networks

Huang, G.-H., see Sun, X.-Y.

Huang, Q. X., see Qiang, S. J.

Huang, S., Zhang, X., Zhang, Y., Hou, S., Yangi, X. \& Zeng, Z., Investigations of the mechanical properties of the $\mathrm{Zr}_{8} \mathrm{Ti}_{8}$ random alloy

Huerta-Quintanilla, R., see Rodríguez-Achach, $\mathrm{M}$.

Hui, P. M., see Li, Y.

Ivanov, A., Novoselov, A. \& Pavlovsky, O., Relativistic path integral Monte Carlo: Relativistic oscillator problem

Jalali, Z. S., Rezvanian, A. \& Meybodi, M. R., Social network sampling using spanning trees

Javarone, M. A., Fermionic networks: Modeling adaptive complex networks with fermionic gases

Jia, B., see Qiang, S. J.

Jia, B., see Zhang, H.-Z.

Jia, X., Hong, J.-S., Gao, Y.-C., Yang, H.-C., Yang, C., Fu, C.-J. \& Hu, J.-Q., Percolation phase transition of static and growing networks under a weighted function
10 (2016) 1650115

7 (2016) 1650076

3 (2016) 1650025

7 (2016) 1650082

2 (2016) 1650019

10 (2016) 1650116

6 (2016) 1650064

8 (2016) 1650085

6 (2016) 1650058

6 (2016) 1650061

7 (2016) 1650076

11 (2016) 1650127

4 (2016) 1650039

11 (2016) 1650133

5 (2016) 1650052

2 (2016) 1650021

6 (2016) 1650061

10 (2016) 1650116

7 (2016) 1650082 
Jian, M.-Y., Shi, J. \& Liu, Y., Analysis of the effect of older drivers' driving behaviors on traffic flow based on a modified $C A$ model

Jiang, R., see Jin, C.-J.

Jiang, R., see Wang, D.-X.

Jiang, R., see Zhang, H.-Z.

Jiang, T., see Liu, F.-M. Jiang, X. S., see Chen, Q. Jiang, Z.-Y., see He, X. Jin, C.-J., Wang, W. \& Jiang, R., Four-phase or two-phase signal plan? A study on four-leg intersection by cellular automaton simulations

Jin, W. W., see Heng, Z.

Jing, Y., see Liu, D.

Juárez-Morales, G., see Benavides-Cruz, M.

Kalmar-Nagy, T., see Zhao, Y.

Kamali, R. \& Manshadi, M. K. D., Numerical simulation of the leaky dielectric microdroplet generation in electric fields

Karmakar, R., Two different modes of oscillation in a gene transcription regulatory network with interlinked positive and negative feedback loops

Karpusenko, V., see Bagli, E.

Kataoka, K., see Zhao, Y.

Katırcioğlu, Ş., see Mohammad, R.

Kaupužs, J., Melnik, R. V. N. \& Rimšāns, J., Corrections to finite-size scaling in the $\varphi^{4}$ model on square lattices

Khansari, M., see Zahedi, R.

Khenata, R., see Labair, M.

Kimura, K. \& Higuchi, S., Monte Carlo estimation of the number of tatami tilings
3 (2016) 1650031

3 (2016) 1650032

2 (2016) 1650019

10 (2016) 1650116

11 (2016) 1650130

6 (2016) 1650063

9 (2016) 1650098

3 (2016) 1650032

1 (2016) 1650005

9 (2016) 1650105

4 (2016) 1650043

7 (2016) 1650077

1 (2016) 1650012

5 (2016) 1650056

2 (2016) 1650022

7 (2016) 1650077

3 (2016) 1650035

9 (2016) 1650108

8 (2016) 1650096

9 (2016) 1650107

11 (2016) 1650128
Ko, B., Song, J. W. \& Chang, W., Simulation of financial market via nonlinear Ising model

Koibuchi, H. \& Shobukhov, A., Internal phase transition induced by external forces in Finsler geometric model for membranes

Kong, Y., see Ren, Z.-M.

Korkmaz, B. \& Dereli, Y., Numerical solution of the rosen a $u-K d V-R L W$ equation by using RBFs collocation method

Krawczyk, M. J. \& Kułakowski, K., Authors as vehicles of scientific memes

Kühne, R. D., see Wang, J.

Kułakowski, K., see Krawczyk, M. J.

Kunwar, A., see Singh, H. P.

Kurniadi, R., Waris, A. \& Viridi, S., Monte Carlo simulation based toy model for fission process

La Rocca, M., see Prestininzi, $\mathrm{P}$

Labair, M., Rached, H., Rached, D., Benalia, S., Abidri, B., Khenata, R., Ahmed, R., Omran, S. B., Bouhemadou, A. \& Syrotyuk, S. V., Prediction of phase transition, mechanical and electronic properties of inverse Heusler compound $Y_{2} R u P b$, via $F P$ LMTO method

4 (2016) 1650038

4 (2016) 1650042

12 (2016) 1650151

10 (2016) 1650117

9 (2016) 1650110

2 (2016) 1650013

9 (2016) 1650110

11 (2016) 1650137

3 (2016) 1650030

4 (2016) 1650037

Lachgar, A. \& Achahbar, A., Network growth with preferential attachment and without "rich get richer" mechanism

Laguna-Camacho, J. R., see Benavides-Cruz, M.

Lakouari, N., see Bentaleb, $\mathrm{K}$.

Lakouari, N., see Echab, H.
9 (2016) 1650107

2 (2016) 1650020

4 (2016) 1650043

2 (2016) 1650023

1 (2016) 1650009 
Author Index

Lamzabi, S., Lazfi, S. Rachadi, A., Ez-Zahraouy, H. \& Benyoussef, A., Modeling the spread of virus in packets on scale free network

Lara-Cisneros, G., see Aguilar-López, R.

Lazfi, S., see Lamzabi, $\mathrm{S}$.

Li, B., see Liu, M.

Li, D., see Han, D.

Li, J.-R., see Zhao, H.-T.

Li, M., see Ma, L.

Li, M., see Zhang, Q.

Li, Q., see Chen, Q.

Li, S. \& He, J., Loss distribution of systemic defaults in different interbank networks

Li, S.-B., see Zhang, L.

Li, S.-N., see Tang, L.-Y.

Li, W., see $\mathrm{Su}, \mathrm{Z}$.

Li, W., see Zhu, Y.

Li, W., see Zou, Y.

Li, X.-G., see Xie, D.-F.

Li, Y., Xu, C., Liu, J. \& Hui, P. M., Competition and time-dependent behavior in spatial Iterated Prisoner's Dilemma incorporating adaptive zerodeterminant strategies

Li, Z., Shi, Y. \& Chen, S., Exploring the influence of human mobility on information spreading in mobile networks

Li, Z., Xu, X., Xu, S., Qian, Y. \& Xu, J., Analytical studies on an extended car following model for mixed traffic flow with slow and fast vehicles

Li, Z., Zhong, C., Chen, L., Xu, S. \& Qian, Y., Analytical studies on a new lattice hydrodynamic traffic flow model with consideration of traffic current cooperation among three consecutive sites

Liang, M., see Yu, Y.
6 (2016) 1650068

4 (2016) 1650040

6 (2016) 1650068

9 (2016) 1650099

6 (2016) 1650057

2 (2016) 1650018

12 (2016) 1650149

10 (2016) 1650118

6 (2016) 1650063

10 (2016) 1650121

12 (2016) 1650145

4 (2016) 1650046

12 (2016) 1650147

10 (2016) 1650122

8 (2016) 1650090

7 (2016) 1650083

4 (2016) 1650039

6 (2016) 1650066

1 (2016) 1650004

3 (2016) 1650034

7 (2016) 1650080
Lichtenegger, K. \& Hadzibeganovic, T., The interplay of self-reflection, social interaction and random events in the dynamics of opinion flow in two-party democracies

Lima, F. W. S., Equilibrium and nonequilibrium models on Solomon networks

Lima, F. W. S., see Radwan, M. A.

Lima, F. W. S., Transition under noise in the Sznajd model on square lattice

Lin, J., see $\mathrm{He}, \mathrm{X}$.

Lin, J.-H., see Tang, L.-Y.

Ling, Y., see Yan, H.

Liu, D., Jing, Y. \& Chang, B., Identifying influential nodes in complex networks based on expansion factor

Liu, D., see Wu, J.

Liu, F.-M., Wang, A.-L., Qiu, R.-F. \& Jiang, T., Improved lattice Boltzmann model for multicomponent diffusion flow with large pressure difference

Liu, J., see Li, Y.

Liu, J.-G., see Tang, L.-Y.

Liu, M. \& Xiong, S., $A$ refined and dynamic cellular automaton model for pedestrian-vehicle mixed traffic flow

Liu, M., Xiong, S. \& Li, B., Dynamic route guidance strategy in a tworoute pedestrian-vehicle mixed traffic flow system Liu, S., Chai, Y., Ding, H. \& Chen, L.-Q., Synchronization of spatiotemporal networks via backstepping using neighbor information

Liu, X., see Yan, W.

Liu, Y., Cheng, R.-J., Ma, Y.-Q. \& Ge, H.-X., The control method for the multi-phase traffic model
5 (2016) 1650065

11 (2016) 1650134

9 (2016) 1650109

3 (2016) 1650026 9 (2016) 1650098 4 (2016) 1650046 10 (2016) 1650115

9 (2016) 1650105 8 (2016) 1650092

11 (2016) 1650130

4 (2016) 1650039

4 (2016) 1650046

5 (2016) 1650053

9 (2016) 1650099

11 (2016) 1650129

8 (2016) 1650086

10 (2016) 1650111 
Liu, Y., see Jian, M.-Y.

Liu, Z., see Yu, Y.

Liu, Z., see Zheng, M.

López-Calderón, J. E., see Benavides-Cruz, M.

López-Pérez, P. A., see Aguilar-López, R.

Lordan, O., see Hong, C.

Lotfi, M., see Enjilela, V.

Luo, S., see Zheng, A.

Lv, R.-L., see Zhang, X.-J.

Ma, J., Han, W., Guo, Q., Wang, Z. \& Zhang, S., A link-adding strategy for transport efficiency of complex networks

Ma, J., Han, W., Guo, Q., Zhang, S., Wang, J. \& Wang, Z., Improved efficient routing strategy on two-layer complex networks

Ma, J., Han, W., Guo, Q. \& Zhang, S., Enhancing traffic capacity of scalefree networks by linkdirected strategy

Ma, J. \& Simos, T. E., Hybrid high algebraic order two-step method with vanished phase-lag and its first, second, third, fourth and fifth derivatives

Ma, L., Zhang, Z. \& Li, M., Surveying traffic congestion based on the concept of community structure of complex networks

Ma, Y.-Q., see Liu, Y.

Mall, U., see Singh, H. P.

Manshadi, M. K. D., see Kamali, R.

Marr, J. H., A novel visualization of the geometry of special relativity

Martín del Rey, A., see Encinas, A. H.

Martín-Vaquero, J., see Encinas, A. H.

Melnik, R. V. N., see Kaupužs, J.

Mendez, M. O., see Alba, A.
3 (2016) 1650031

7 (2016) 1650080

5 (2016) 1650048

4 (2016) 1650043

4 (2016) 1650040

11 (2016) 1650131

7 (2016) 1650081

1 (2016) 1650002

8 (2016) 1650093

5 (2016) 1650054

4 (2016) 1650044

3 (2016) 1650028

5 (2016) 1650049

12 (2016) 1650149

10 (2016) 1650111

11 (2016) 1650137

1 (2016) 1650012

5 (2016) 1650055

9 (2016) 1650097

9 (2016) 1650097

9 (2016) 1650108

3 (2016) 1650029
Mendonça, J. R. G., The inactive-active phase transition in the noisy additive (exclusive-or) probabilistic cellular automaton

2 (2016) 1650016

Mesri, Y., see Deyranlou, A.

1 (2016) 1650003

Meybodi, M. R., see Jalali, Z. S.

Milioli, G., see ReyesSanchez, E.

Miranda, J. G. V., see Azevedo, S. M.

Méndez, M. O., see De León-Lomelí, R.

Méndez, M. O., see ReyesSanchez, E.

Modanese, G., see Bertotti, M. L.

Mohammad, R. \& Katırc1oğlu, Ş., First-principles calculations for the structural and electronic properties of GaAs $_{1-x} P_{x}$ nanowires

Molina, J. M., see Sergi, D.

Montessori, A., see Prestininzi, $\mathrm{P}$

Morales-Mendoza, L. J., see Benavides-Cruz, M.

Moreira, A. A., see de Brito, J. B.

Moret, M. A., see Azevedo, S. M.

Moukarzel, C. F., see Bustos-Guajardo, R.

Murguía, J. S., see De León-Lomelí, R.

Myrheim, J., see Hopstad, Y.

Nagler, J., see Verma, $\mathrm{T}$.

Narciso, J., see Sergi, D.

Nestola, M. G. C., Gizzi, A., Cherubini, C. \& Filippi, S., Three-band decomposition analysis in multiscale FSI models of abdominal aortic aneurysms

$\mathrm{Ni}, \mathrm{S}$., see Wu, J.

Niazmand, H., see Deyranlou, A.
3 (2016) 1650035

6 (2016) 1650062

5 (2016) 1650052

12 (2016) 1650140

12 (2016) 1650143

7 (2016) 1650071

12 (2016) 1650140

9 (2016) 1650100

4 (2016) 1650037

4 (2016) 1650043

3 (2016) 1650024

12 (2016) 1650143

8 (2016) 1650094

7 (2016) 1650071

12 (2016) 1650142

12 (2016) 1650141

6 (2016) 1650062

2 (2016) 1650017

7 (2016) 1650079

1 (2016) 1650003 
Nie, C., see Zhao, H.-T.

Niu, K., see He, X.

Noorani, M. S. Md, see Vahedi, S.

Novoselov, A., see Ivanov, A.

Olivares-Peregrino, V. H., see Gómez-Aguilar, J. F.

Omran, S. B., see Labair, M.

Orlik, P., see Zhao, Y.

Ortona, A., see Sergi, D.

Oruç, Ö., Esen, A. \& Bulut, F., A Haar wavelet collocation method for coupled nonlinear Schrödinger-KdV equations

Oświęcimka, P., see Górski, A. Z.

Parrino, L., see ReyesSanchez, E.

Parsons, K., see Zhao, Y. Pasini, P., see Chiccoli, C. Passos, P., see Gama, J.

Pavlovsky, O., see Ivanov, A.

Peng, G., The effect of interruption probability in lattice model of twolane traffic flow with passing

Pengjun, Z., Wei, W. \& Hongxia, G., The influence of bus stop on traffic flow with velocity-difference-separation model

Pérez-Meana, H., see Benavides-Cruz, M.

Prestininzi, P., Montessori, A., La Rocca, M. \& Succi, S., Reassessing the single relaxation time Lattice Boltzmann method for the simulation of Darcy's flows

Qian, Y., see Li, Z.

Qian, Y., see Li, Z.

Qiang, S. J., Jia, B., Huang, Q. X. \& Gao, Z. Y., Mechanism behind phase transitions in airplane boarding process

Qiu, R.-F., see Liu, F.-M.

Queiruga-Dios, A., see Encinas, A. H.
2 (2016) 1650018

9 (2016) 1650098

8 (2016) 1650087

11 (2016) 1650133

1 (2016) 1650007

9 (2016) 1650107

7 (2016) 1650077

6 (2016) 1650062

9 (2016) 1650103

10 (2016) 1650112

12 (2016) 1650140

7 (2016) 1650077

10 (2016) 1650114

9 (2016) 1650102

11 (2016) 1650133

5 (2016) 1650050

11 (2016) 1650135

4 (2016) 1650043

4 (2016) 1650037

1 (2016) 1650004

3 (2016) 1650034

6 (2016) 1650061

11 (2016) 1650130

9 (2016) 1650097
Rachadi, A., see Lamzabi, $\mathrm{S}$.

Rached, D., see Labair, M.

6 (2016) 1650068

9 (2016) 1650107

Rached, H., see Labair, M.

9 (2016) 1650107

Radwan, M. A., Sumour, M. A., Elbitar, A. M., Shabat, M. M. \& Lima, F. W. S., Finite-size effects on semi-directed Barabási-Albert networks

Rahali, G., see Hashemloo, A.

Rao, P., see Xu, L.

Re Fiorentin, M., FaRe: A Mathematica package for tensor reduction of Feynman integrals

Ren, J., see Huang, G.

Ren, L., see Gao, X.

Ren, Z.-M. \& Kong, Y., Coarse-graining the input of education and $R \& D$ in China

Reyes-Sanchez, E., Alba, A., Méndez, M. O., Milioli, G. \& Parrino, L., Spectral entropy analysis of the respiratory signal and its relationship with the cyclic alternating pattern during sleep

Rezvanian, A., see Jalali, Z. S.

Ribeiro, J., see Gama, J.

Rimšāns, J., see Kaupužs, J.

Rodríguez-Achach, M., Coronel-Brizio, H. F., Hernández-Montoya, A. R., Huerta-Quintanilla, R. \& CantoLugo, E., The onedimensional minesweeper game: What are your chances of winning?

Rodríguez-Achach, M., see Benavides-Cruz, M.

Rong, L.-L., see Yan, K.-S. Ruan, Z., see Zheng, M.

Rubio-Rincon, M. E., see Alba, A.
9 (2016) 1650109

2 (2016) 1650014

12 (2016) 1650144

3 (2016) 1650027

8 (2016) 1650085

11 (2016) 1650138

12 (2016) 1650151

12 (2016) 1650140

5 (2016) 1650052

9 (2016) 1650102

9 (2016) 1650108

11 (2016) 1650127

4 (2016) 1650043

5 (2016) 1650051

5 (2016) 1650048

3 (2016) 1650029 
Ruivo, E. L. P. \& De Oliveira, P. P. B., Computing cellular automata spectra under fixed boundary conditions via limit graphs

Saba, H., see Azevedo, S. M.

Sadeghi, M.-R., see Deyranlou, A.

Saghatchi, R., see Ghazanfarian, J.

Saha Ray, S., see Sahoo, S.

Sahoo, S., Saha Ray, S., Das, S. \& Bera, R. K., The formation of dynamic variable order fractional differential equation

Sak-Saracino, E. \& Urbassek, H. M., The $\alpha \leftrightarrow \gamma$ transformation of an $\mathrm{Fe}_{1-x} \mathrm{Cr}_{x}$ alloy: $\mathrm{A}$ molecular-dynamics approach

Salehi, S. \& Taghiyareh, F., Introducing a more realistic model for opinion formation considering instability in social structure

Salimi, D., see Enjilela, V.

Sallan, J. M., see Hong, C.

Sampaio Filho, C. I. N., see de Brito, J. B.

Schaefer, L., see $\mathrm{Xu}, \mathrm{L}$.

Seixas, J. M., see Simas Filho, E. F.

Sergi, D., Camarano, A., Molina, J. M., Ortona, A. \& Narciso, J., Surface growth for molten silicon infiltration into carbon millimeter-sized channels: Lattice-Boltzmann simulations, experiments and models

Seyrich, M. \& Sornette, D., Micro-foundation using percolation theory of the finite time singular behavior of the crash hazard rate in a class of rational expectation bubbles

Shabat, M. M., see Radwan, M. A.

Shang, P., see Gao, X.

\section{7 (2016) 1650073 \\ 12 (2016) 1650143}

1 (2016) 1650003

12 (2016) 1650152

7 (2016) 1650074

7 (2016) 1650074

10 (2016) 1650124

11 (2016) 1650136 7 (2016) 1650081 11 (2016) 1650131

3 (2016) 1650024

12 (2016) 1650144

5 (2016) 1630002

6 (2016) 1650062

10 (2016) 1650113

9 (2016) 1650109

11 (2016) 1650138
Shen, H., see $\mathrm{Wu}, \mathrm{B}$.

Shen, J., see Huang, A.

Shen, S., see $\mathrm{Wu}$, J.

Shi, J., see Jian, M.-Y.

Shi, Q., see Wang, J.

Shi, Y., see $\mathrm{Li}, \mathrm{Z}$.

Shi, Z., see Yan, F.

Shi, Z.-K., see Zhou, J.

Shobukhov, A., see Koibuchi, $\mathrm{H}$.

Simas Filho, E. F. \& Seixas, J. M., Unsupervised statistical learning applied to experimental high-energy physics and related areas

Simos, T. E., see Ma, J.

Singh, H. P., Takshak, A., Mall, U. \& Kunwar, A., Sliding of microtubules by a team of dynein motors: Understanding the effect of spatial distribution of motor tails and mutual exclusion of motor heads on microtubules

Skrzat, J., see Górski, A. $Z$.

Sochi, T., Deterministic and stochastic algorithms for resolving the flow fields in ducts and networks using energy minimization

Song, J. W., see Ko, B.

Sornette, D., see Seyrich, M.

Stauffer, D., Income inequality in the 21st century - A biased summary of Piketty's capital in the twenty-first century

Stróż, M., see Górski, A. Z.

Su, Z., Deng, W., Han, J., Li, W. \& Cai, X., Occurrence of synchronized flow due to overtaking strategy in the nagelSchreckenberg model

Succi, S., see Prestininzi, P.

Sumour, M. A., see Radwan, M. A.

Sun, M., see Han, D.
7 (2016) 1650072

6 (2016) 1650064

7 (2016) 1650079

3 (2016) 1650031

2 (2016) 1650013

6 (2016) 1650066

4 (2016) 1650045

6 (2016) 1650069

4 (2016) 1650042

5 (2016) 1630002

5 (2016) 1650049

11 (2016) 1650137

10 (2016) 1650112

4 (2016) 1650036

4 (2016) 1650038

10 (2016) 1650113

2 (2016) 1630001

10 (2016) 1650112

12 (2016) 1650147

4 (2016) 1650037

9 (2016) 1650109

6 (2016) 1650057 
Sun, X.-Y., Ding, Z.-J. \& Huang, G.-H., Effect of density feedback on the two-route traffic scenario with bottleneck

Syrotyuk, S. V., see Labair, M.

Taghiyareh, F., see Salehi, $\mathrm{S}$

Takshak, A., see Singh, H. P.

Tan, F., see Zhang, W.-P. Tang, L.-Y., Li, S.-N., Lin, J.-H., Guo, Q. \& Liu, J.-G., Community structure detection based on the neighbor node degree information

Tang, M., see Zheng, M.

Tang, T.-Q. \& Yu, Q., Influences of vehicles fuel consumption and exhaust emissions on the trip cost without late arrival under car-following model

Taran, S., Garip, A. K. \& Arslan, H., Theoretical study of the structures and chemical ordering of $\mathrm{CoPd}$ nanoalloys supported on $\mathrm{MgO}(001)$

Tavasoli, A., see Enjilela, V. Tian, F., see Yan, F.

Torreão, J. R. A., Holonomy, quantum mechanics and the signal-tuned Gabor approach to the striate cortex

Urbassek, H. M., see Sak-Saracino, E.

Vahedi, S. \& Noorani, M. $\mathrm{S}$. Md, Cluster modified projective synchronization between networks with distinct topologies

Verma, T., Araújo, N. A M., Nagler, J., Andrade, Jr. J. S. \& Herrmann, H. J., Model for the growth of the world airline network

Viridi, S., see Kurniadi, $\mathrm{R}$.

Wang, A.-L., see Liu, F.-M.
6 (2016) 1650058

9 (2016) 1650107

11 (2016) 1650136

11 (2016) 1650137

6 (2016) 1650059

4 (2016) 1650046

5 (2016) 1650048

1 (2016) 1650011

12 (2016) 1650146

7 (2016) 1650081

4 (2016) 1650045

8 (2016) 1650089

10 (2016) 1650124

8 (2016) 1650087

12 (2016) 1650141

3 (2016) 1650030

11 (2016) 1650130
Wang, B.-H., see Yang, H.-X.

Wang, D., see Zhou, Z.-C.

Wang, D.-X., Jiang, R., $\mathrm{Hu}$, M.-B. \& Wu, Q.-S., Power spectra of totally asymmetric exclusion processes on lattices with a junction

Wang, J., Ding, J.-X., Shi, Q. \& Kühne, R. D., Lane-changing behavior and its effect on energy dissipation using full velocity difference model

Wang, J., see Ma, J.

Wang, K., see Guan, J.

Wang, Q. A., see Zhu, Y.

Wang, W., see Jin, C.-J.

Wang, X., see Wei, N.

Wang, Y. \& Xu, X.-J., Quantum transport with long-range steps on Watts-Strogatz networks

Wang, Z., see Ma, J.

Wang, Z., see Ma, J.

Waris, A., see Kurniadi, R.

Wei, N., Wang, X. \& Zuo, $\mathrm{X}$., The mechanical and thermodynamic properties of $\mathrm{ZrAl}_{2}$ under pressure from first-principles investigation

1 (2016) 1650001

11 (2016) 1650135

8 (2016) 1650086

2 (2016) 1650018

Wei, Y.-A., see Zhao, H.-T.

$\mathrm{Wu}$, B. \& Shen, H., Mining connected global and local dense subgraphs for bigdata

$\mathrm{Wu}, \mathrm{G}$., see Yan, $\mathrm{H}$.

Wu, J., Du, R., Zheng, Y. \& Liu, D., Optimal multi-community network modularity for information diffusion

2 (2016) 1650013 4 (2016) 1650044 9 (2016) 1650104 10 (2016) 1650122 3 (2016) 1650032 1 (2016) 1650001

2 (2016) 1650015 4 (2016) 1650044 5 (2016) 1650054

3 (2016) 1650030

7 (2016) 1650072

10 (2016) 1650115

8 (2016) 1650092

Wu, J., Ni, S. \& Shen, S., Dynamics of public opinion under the influence of epidemic spreading

$\mathrm{Wu}, \mathrm{J}$., see Yan, W.

$\mathrm{Wu}$, Q.-S., see Wang, D.-X.

Wu, Z., see Gong, X.
7 (2016) 1650079

8 (2016) 1650086

2 (2016) 1650019

1 (2016) 1650010 
Xamán, J., see ZavalaGuillén, I.

Xia, H., see Zheng, A.

Xia, Y., see Zhang, W.-P.

Xiao, X., see Heng, Z.

Xiao, Y., see Zhou, Z.-C.

Xie, D.-F., Zhao, X.-M., Li, X.-G. \& Zhu, T.-L., Modeling the impact of pedestrian behavior diversity on traffic dynamics at a crosswalk with push button

Xiong, J., see Huang, A.

Xiong, S., see Liu, M.

Xiong, S., see Liu, M.

$\mathrm{Xu}, \mathrm{C}$., see Li, Y.

$\mathrm{Xu}$, J., see $\mathrm{Li}, \mathrm{Z}$.

$\mathrm{Xu}, \mathrm{L} ., \mathrm{Rao}, \mathrm{P}$. \& Schaefer, L., A novel scheme for curved moving boundaries in the lattice Boltzmann method

$\mathrm{Xu}, \mathrm{P}$., see Yan, W.

$\mathrm{Xu}, \mathrm{S}$., see $\mathrm{Li}, \mathrm{Z}$.

$\mathrm{Xu}, \mathrm{S}$., see Li, Z.

$\mathrm{Xu}, \mathrm{X}$., see $\mathrm{Li}, \mathrm{Z}$.

$\mathrm{Xu}, \mathrm{X}$.-J., see Wang, Y.

Yan, F., Tian, F. \& Shi, Z., Effects of iterative learning based signal control strategies on macroscopic fundamental diagrams of urban road networks

Yan, H., Hou, L., Ling, Y. \& Wu, G., Optimizing complex networks controllability by local structure information

Yan, K.-S., Rong, L.-L. \& $\mathrm{Yu}, \mathrm{K}$., Discriminating complex networks through supervised NDR and Bayesian classifier

Yan, W., Liu, X., Wu, J., Wei, Y. \& Xu, P., Numerical simulation on the reduction of flow heterogeneity in the biofilter media

Yang, C., see Jia, X.

Yang, H.-C., see Jia, X.
8 (2016) 1650095

1 (2016) 1650002

6 (2016) 1650059

1 (2016) 1650005

7 (2016) 1650078

7 (2016) 1650083

6 (2016) 1650064

5 (2016) 1650053

9 (2016) 1650099

4 (2016) 1650039

1 (2016) 1650004

12 (2016) 1650144

8 (2016) 1650086

1 (2016) 1650004

3 (2016) 1650034

1 (2016) 1650004

2 (2016) 1650015

4 (2016) 1650045

10 (2016) 1650115

5 (2016) 1650051

8 (2016) 1650086

7 (2016) 1650082

7 (2016) 1650082
Yang, H.-X. \& Wang, B.H., Traffic-driven epidemic spreading on scale-free networks with tunable degree distribution

11 (2016) 1650125

Yangi, X., see Huang, S.

Yevick, D., Accelerated rare event sampling

Yevick, D., Renormalized multicanonical sampling

Yi, H., see Heng, Z.

Yin, T., see Huang, G.

Youqin, W., see Heng, $\mathrm{Z}$.

$\mathrm{Yu}, \mathrm{K}$., see Yan, K.-S.

$\mathrm{Yu}, \mathrm{Q}$., see Tang, T.-Q.

$\mathrm{Yu}, \mathrm{Y}$., Liang, M. \& Liu, Z., An optimized nodedisjoint multipath routing scheme in mobile ad hoc

Zadehgol, A. \& Ashrafizaadeh, M., A multiple relaxation time extension of the constant speed kinetic model

Zahedi, R. \& Khansari, M., Network immunization under limited budget using graph spectra

Zannoni, C., see Chiccoli, C.

Zarei, M., see Zarei, M.

Zarei, M., Zarei, M. \& Hashemian, M., Effect of phenolic radicals on the geometry and electronic structure of DNA base pairs: Computational study

Zavala-Guillén, I., Xamán, J., Álvarez, G., Arce, J., HernándezPérez, I. \& Gijón-Rivera, M., Computational fluid dynamics for modeling the turbulent natural convection in a double air-channel solar chimney system

Zeng, A., see Zhang, C.-J. Zeng, Z., see Huang, S.
7 (2016) 1650076

4 (2016) 1650041

3 (2016) 1650033

1 (2016) 1650005

8 (2016) 1650085

5 (2016) 1650051

1 (2016) 1650011

7 (2016) 1650080

8 (2016) 1650088

8 (2016) 1650096

10 (2016) 1650114

10 (2016) 1650119

10 (2016) 1650119

8 (2016) 1650095

10 (2016) 1650120

7 (2016) 1650076
1 (2016) 1650005 
Zhang, B., see Huang, G. Zhang, C.-J. \& Zeng, A., Prediction of missing links and reconstruction of complex networks

Zhang, H., see $\mathrm{He}, \mathrm{X}$.

Zhang, H.-Z., Jiang, R., Hu, M.-B. \& Jia, B., Analytical investigation on the minimum traffic delay at a two-phase intersection considering the dynamical evolution process of queues

Zhang, J., see Hong, C.

Zhang, L., Fu, B.-B. \& Li, S.-B., Cascading failures coupled model of interdependent double layered public transit network

Zhang, M., see Han, M.

Zhang, P., see Huang, G.

Zhang, Q., Li, M. \& Deng, Y., A new structure entropy of complex networks based on nonextensive statistical mechanics

Zhang, S., see Ma, J.

Zhang, S., see Ma, J.

Zhang, S., see Ma, J.

Zhang, W.-P., Xia, Y. \& Tan, F., Oscillations in interconnected complex networks under intentional attack

Zhang, X., see Huang, S.

Zhang, X. B., see Chen, Q.

Zhang, X.-J., Gu, B., Guan, X.-M., Zhu, Y.-B. \& Lv, R.-L., Cascading failure in scale-free networks with tunable clustering

Zhang, Y., see Han, M.

Zhang, Y., see Huang, S.

Zhang, Z., see Ma, L.

Zhao, H.-T., Nie, C., Li, J.-R. \& Wei, Y.-A., $A$ two-lane cellular automaton traffic flow model with the influence of driver, vehicle and road
8 (2016) 1650085

10 (2016) 1650120

9 (2016) 1650098

10 (2016) 1650116

11 (2016) 1650131

12 (2016) 1650145

11 (2016) 1650126

8 (2016) 1650085

10 (2016) 1650118

3 (2016) 1650028

4 (2016) 1650044

5 (2016) 1650054

6 (2016) 1650059

7 (2016) 1650076

6 (2016) 1650063

8 (2016) 1650093

11 (2016) 1650126

7 (2016) 1650076

12 (2016) 1650149

2 (2016) 1650018
Zhao, L., see Zhu, Y.

Zhao, X.-M., see Xie, D.-F.

Zhao, Y., Orlik, P., Parsons, K., Kataoka, K. \& $\mathrm{Kalmar}-\mathrm{Nagy}$, T., Improvement of train transportation performance by convoy signaling

Zheng, A., Luo, S. \& Xia, H., A meme propagation model to combine social affirmation with meme attractiveness and persistence

Zheng, M., Ruan, Z., Tang, M., Do, Y. \& Liu, Z., Influence of periodic traffic congestion on epidemic spreading

Zheng, Y., see Wu, J.

Zhong, C., see Li, Z.

Zhou, H. P., see Chen, Q.

Zhou, J. \& Shi, Z.-K., A modified full velocity difference model with the consideration of velocity deviation

Zhou, Z.-C., Xiao, Y. \& Wang, D., Constraints of nonresponding flows based on cross layers in the networks

Zhu, T.-L., see Xie, D.-F.

Zhu, Y., Zhao, L., Li, W., Wang, Q. A. \& Cai, X., Random walks on real metro systems

Zhu, Y.-B., see Zhang, X.-J.

Zou, Y., Deng, W., Li, W. \& Cai, X., A study of epidemic spreading on activity-driven networks Zuo, X., see Wei, N.
10 (2016) 1650122

7 (2016) 1650083

7 (2016) 1650077

1 (2016) 1650002

5 (2016) 1650048

8 (2016) 1650092

3 (2016) 1650034

6 (2016) 1650063

6 (2016) 1650069

7 (2016) 1650078

7 (2016) 1650083

10 (2016) 1650122

8 (2016) 1650093

8 (2016) 1650090 1 (2016) 1650001 